\title{
VNT Based Distribution Automation System
}

\author{
Prachi Yadav ${ }^{1}$, Nisheet Soni ${ }^{2}$ \\ ${ }^{I}$ (Electrical Department, Shriram Institute of Technology/ RGPV, India) \\ ${ }^{2}$ (Electrical Department, Shriram Institute of Technology/ RGPV, India)
}

\begin{abstract}
Despite years of activity, truly open and intelligent control systems seem still to be a promise of the future. Agreement on common architectures and application objects is needed to raise open control systems from exchanging raw data to the level of real interoperability of off-the-shelf components. Future control platforms and programming languages should have new built-in mechanisms that support implementation of intelligent functions, such as flexible resource management and exception handling. This article argues that many of these challenges can be met by taking full advantage of emerging software engineering technologies. This also means that the modeling techniques and design practices of software engineering should be combined with the traditional ways of thinking in distribution automation.

This paper proposes a distribution automation system based on vehicular network toolbox incorporated in Matlab release 2011. Based on it voltages and current of 11 distribution transformers with a graphical user interface is proposed.
\end{abstract}

Keywords: Distribution Automation, VNT, GUI.

\section{INTRODUCTION}

Electrical energy demand is ever increasing. Electric power is normally generated at $11-25 \mathrm{kV}$ in a power station. To transmit over long distances, it is then stepped-up to $400 \mathrm{kV}, 220 \mathrm{kV}$ or $132 \mathrm{kV}$ as necessary. Power is carried through a transmission network of high voltage lines. Usually, these lines run into hundreds of kilometers and deliver the power into a common power pool called the grid. The grid is connected to load centers (cities) through a sub-transmission network of normally $33 \mathrm{kV}$ (or sometimes $66 \mathrm{kV}$ ) lines. These lines terminate into a $33 \mathrm{kV}$ (or $66 \mathrm{kV}$ ) substation, where the voltage is stepped-down to $11 \mathrm{kV}$ for power distribution to load points through a distribution network of lines at $11 \mathrm{kV}$ and lower.

Today over $21 \%$ of the total electrical energy generated in India is lost in transmission and distribution. The electrical power deficit in the country is currently about $18 \%$. Clearly, reduction in distribution losses can reduce this deficit significantly. It is possible to bring down the distribution losses to a 6-8 \% level in India with the help of newer technological options in the electrical power distribution sector which will enable better monitoring and control. The power network, which generally concerns the common man, is the distribution network of $11 \mathrm{kV}$ lines or feeders downstream of the $33 \mathrm{kV}$ substation. Each $11 \mathrm{kV}$ feeder which emanates from the $33 \mathrm{kV}$ substation branches further into several subsidiary $11 \mathrm{kV}$ feeders to carry power close to the load points (localities, industrial areas, villages, etc.,). At these load points, a transformer further reduces the voltage from $11 \mathrm{kV}$ to $415 \mathrm{~V}$ to provide the last-mile connection through $415 \mathrm{~V}$ feeders (also called as Low Tension (LT) feeders) to individual customers, either at $240 \mathrm{~V}$ (as single-phase supply) or at $415 \mathrm{~V}$ (as three-phase supply). A feeder could be either an overhead line or an underground cable. In urban areas, owing to the density of customers, the length of an $11 \mathrm{kV}$ feeder is generally up to $3 \mathrm{~km}$. On the other hand, in rural areas, the feeder length is much larger (up to $20 \mathrm{~km}$ ). A $415 \mathrm{~V}$ feeder should normally be restricted to about $0.5-1.0 \mathrm{~km}$. unduly long feeder's lead to low voltage at the consumer end. 


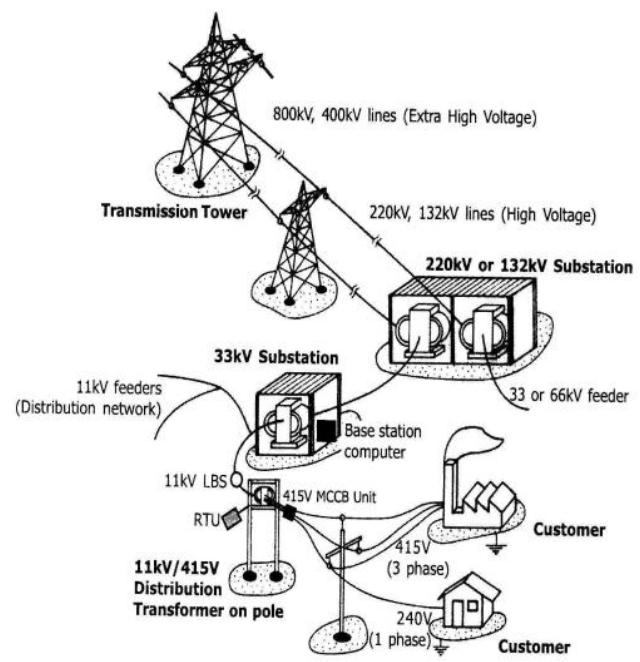

Fig. 1 Basic Distribution Structure

\subsection{Power Line Communication}

\section{CURRENT TRENDS IN DISRIBUTION AUTOMATION}

The first DAS using PLC was installed in Kyong-Gi District Head Office in 1983. This system uses two-way data transmission over the $22.9 \mathrm{kV}$ distribution network. At the substation, signals are injected onto the $22.9 \mathrm{kV}$ distribution network and 42 automatic switches in two substations have been remotely controlled. However, the signal transmission speed was too slow compared with other communication media. In the case of frequent changes of distribution lines like underground construction and load transfer, the change of communication path often causes transmission failure, which is called "open circuit problem." In other words, communication is lost with devices on the far side of an open circuit. This severely restricts the usefulness of PLC systems for applications involving reclosers, switches, sectionalizes, and outage detection. PLC systems also require that a signal transmitter/receiver be installed in all distribution substations that have a downstream PLC device. These are expensive and can have significant negative impact on the cost effectiveness of PLC solutions. Recently, a new technology using high frequencies is being developed and under field test, with the advantage that utilities do not need to invest for additional communication network.

\subsection{Pair-Cable}

The prototype of Korean DAS (KODAS) was developed jointly by KEPCO Research Institute (KEPRI), Korea Electro technology Research Institute and six industrial partners from 1991 to 1993, as a national research project to enhance the competitiveness of domestic manufacturing industry. To evaluate in the real field, the prototype was installed in Kang-Dong Branch Office (B/O) in Seoul and in operation since 1994. Asa communication media, $70 \mathrm{~km}$-long pair cables were constructed and 125 automatic switches were installed to verify theremote control functions. The other SCADA system using pair-cable has been in operation to study its performance and applicability to distribution line. The 122 automatic switches in $22 \mathrm{kV}$ - underground distribution lines have been remotely controlled in Choong-Boo B/O in downtown Seoul. Due to the low efficiency and poor reliability, pair cable has not been extended any more.

\subsection{Telephone Line}

The demonstration system using telephone line was installed in Ul-ReungB/O in 1997.Upon evaluation of the model system, it has been expanded to eight B/Os in rural area since 1998and recently, and about $19 \%$ of automatic switches have been controlled by telephone line. The advantage of telephone line is its wide service area all over the country. However, different ownership of the line and equipment's often cause the delay of service restoration. Some intermittent communication errors still need to be improved continuously. These days, telephone lines are used less because of the costs associated with installation of telephone lines and dielectric isolation equipment, and also due to the monthly cost. The cost for installation of telephone lines is increasing with the remoteness of the location.

\subsection{Wireless Data Communication}

Wireless solutions have shown the greatest potential for automating distribution networks because they communicate virtually anywhere at a very low cost. A demonstration system using a private wireless network 
was developed and installed in Gyong-GiB/O to test the feasibility of data communication. Upon evaluation of the model system, DA using wireless data service has been expanded in their possible service areas. However, the service area of wireless data communication network is restricted to some big cities for their commercial use, and data communication response had been delayed during peak time intervals like an opening time of the stock exchange market.

\subsection{Fiber-Optic Cable}

Fiber-optic cable is a very technically attractive solution, offering relatively unlimited bandwidth. Its dielectric and EMI/RFI noise immunity characteristics make it an ideal fit in the high-voltage operating environment. While fiber optic solutions are expensive, they offer two large benefits: first it allows utilities to bring back large amount of data on a frequent basis. Second, it can provide true, real-time communications. These benefits make fiber optic communications an attractive alternative if getting large amounts of data on a real time basis is critical and the location is not extremely remote. KEPCO itself possesses a huge backbone network covering the GW (composite ground wire with optical fiber). Large-scale D whole country with OPAS in urban area needs high reliability and high-speed because it needs to process a large amount of data in a short time compared with small-scale DAS in rural area with more dispersed facilities. About $57 \%$ of automatic switches in large cities have been remotely controlled, showing the best reliability and communication speed among all other communication media.

\section{VEHICULAR NETWORK TOOLBOX}

Vehicle Network Toolbox ${ }^{\mathrm{TM}}$ provides connectivity to CAN devices from MATLAB ${ }^{\circledR}$ and Simulink ${ }^{\circledR}$ using industry-standard CAN database files. The toolbox provides MATLAB functions and Simulink blocks to send, receive, encode, and decode CAN and XCP messages, enabling you to exchange messages between a CAN bus and your programs and models. You also can connect to an ECU via XCP on CAN using A2L description files. From MATLAB or Simulink, you can monitor, filter, and analyze live CAN bus data or log and record CAN messages for later analysis and replay. You also can simulate message traffic on a virtual CAN bus or connect Simulink models to a live network or ECU. Vehicle Network Toolbox supports CAN interface devices from Vector, Kvaser, and National Instruments ${ }^{\circledR}$.

Fig below shows a representation of a typical CAN workflow in Matlab.

Key Features of VNT are as below:-

- MATLAB functions for transmitting and receiving

- CAN and XCP messages Simulink CAN and XCP blocks for connecting a model to a CAN bus or ECUVector CAN database (.dbc) file and A2L description support

- Signal packing and unpacking functions and blocks for simplified encoding and decoding of CAN messages

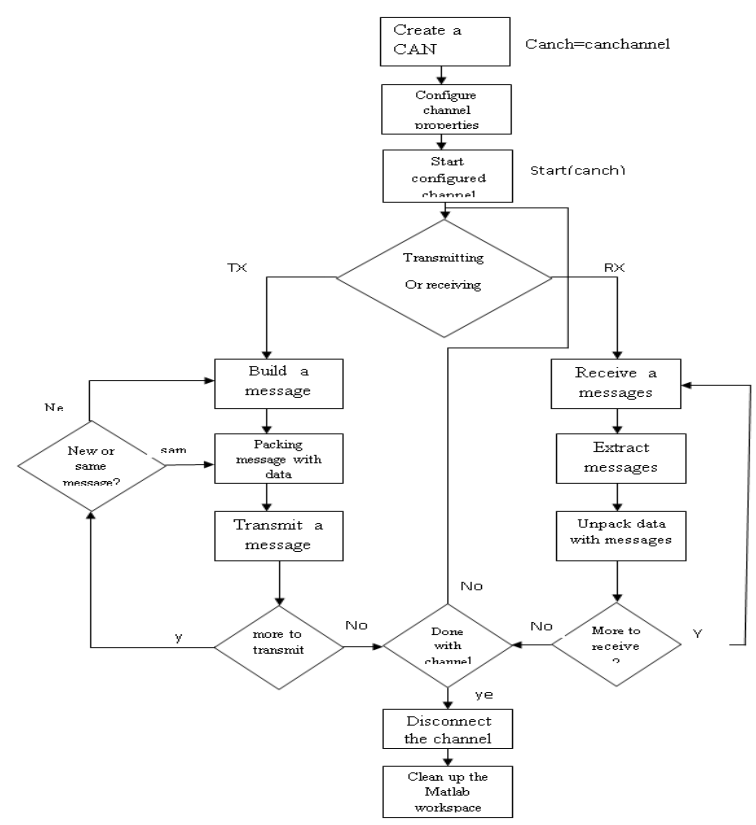

Fig. 2 Basic CAN workflow in VNT 


\section{USING CONTROL AREA NETWORK FOR DA SYSTEM}

Controller Area Network or so-called CAN is a serial bus that utilizes broadcast method to transmit messages across all CAN nodes. It uses a serial control protocol which provides reliable, efficient and economic link between devices to support the distributed real time applications by using a bitwise deterministic collisionresolution mechanism. It was originally developed in the 1980s by Robert Boush as an alternative data communications for interconnecting the control components in automotive vehicles. Prior to CAN technology, all manufacturers used to connect devices within vehicles using point to point wiring systems. Wiring started to become more complex, bulky, heavy and expensive as more electronics and controllers are deployed in a vehicle. This problem can be seen in Figure 1(a), where the abundance of wiring is required which makes the whole circuit even more complicated. CAN system can solve this problem by utilizing a twisted pair cable to communicate with each other as shown in Figure 1(b).Initially, it was designed to allow the microcontrollers and devices to communicate with each other within a vehicle without a host computer. It has been fast gaining wide appreciation with further applied in various automation industrial including military, aviation, electronics, factories and many others due to its high immunity towards electrical interference, and the ability to selfdiagnose and repair the data errors. Additionally, the low cost, performance and upgradeability to provide tremendous flexibility in the system design add to its many advantages.

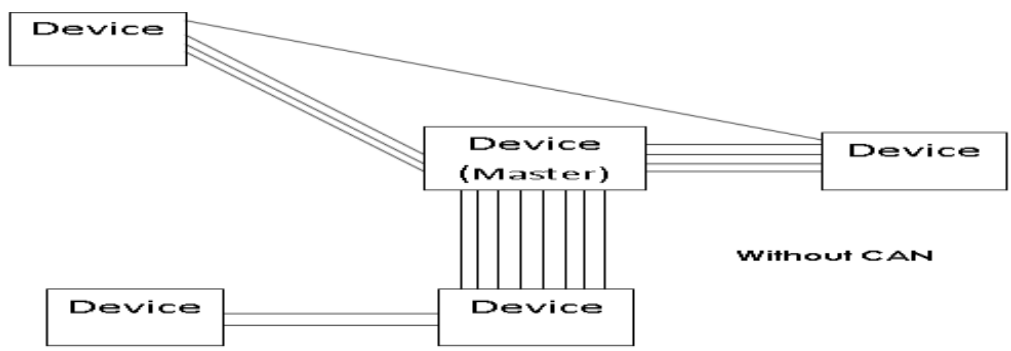

Fig. 2 (a) Traditional Wiring

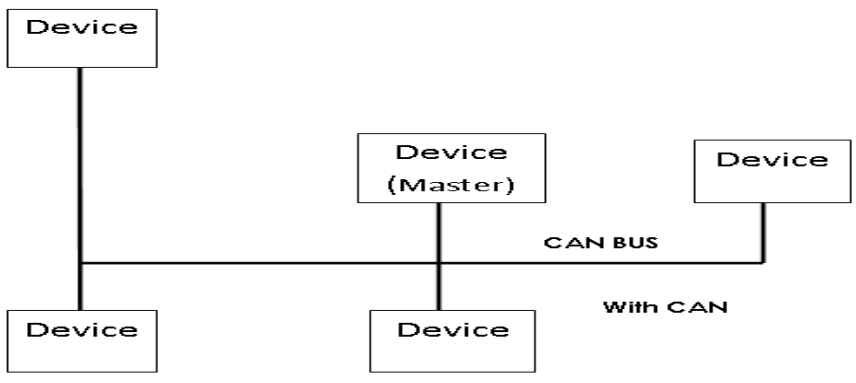

Fig. 2 (b) CAN Wiring

\section{CAN PROTOCOL}

The CAN system uses carrier sense multiple access with collision detection (CSMA/CD) and arbitration on message priority as its communication protocol. This communication protocol allows every node in CAN to monitor the network bus in advance before attempting to transmit a message.

When no activity occurs in the network, each node has the same opportunity to transmit a message. Additionally, this communication protocol allows collision to be solved by using bit-wise arbitration. It is based on a pre-programmed priority of each message in the identifier field of a message. This configuration allows the messages to remain intact after the arbitration is completed even if collisions are detected. In order for the arbitration process to be successful, the logic states need to be defined as dominant or recessive. An example of CAN arbitration can be seen when three nodes are assumed to be transmitting simultaneously.

When three nodes start transmitting their start of frame(SOF) bits simultaneously, the Nodes 1 and 2 stop transmitting as soon as they transmit bit ' 1 ' (recessive level) while Node 3 is transmitting bit ' 0 ' (dominant level). At this instance, Node 3 will continue its transmission when the identifier of bit ' 0 ' has been transmitted while Nodes 1 and 2 are entering into the receiver mode which indicated in grey color. The CAN protocol is defined with the ISO standard of 11-bit identifier that provides for the signaling rates from $125 \mathrm{kbps}$ to $1 \mathrm{Mbps}$. This standard is later improved to allow for larger number of bit with the "extended" version of 29-bit identifier. The 11-bit identifier standard provides 211 or 2048 different message identifiers while the extended 29-bit identifier standard provides 229 or 537 million identifiers [9]. 


\section{CAN COMMUNICATION IN MATLAB}

Vehicle Network Toolbox ${ }^{\mathrm{TM}}$ provides connectivity to CAN devices from MATLAB ${ }^{\circledR}$ and Simulink ${ }^{\circledR}$ using industry-standard CAN database files. The toolbox provides MATLAB functions and Simulink blocks to send, receive, encode, and decode CAN and XCP messages, enabling you to exchange messages between a CAN bus and your programs and models. It also can connect to an ECU via XCP on CAN using A2L description files.

From MATLAB or Simulink, we can monitor, filter, and analyze live CAN bus data or log and record CAN messages for later analysis and replay. Also simulate message traffic on a virtual CAN bus or connect Simulink models to a live network or ECU. Vehicle Network Toolbox supports CAN interface devices from Vector, Kvaser, and National Instruments.

\section{PROPOSED DA SYSTEM}

The proposed system will incorporate all the features of CAN communication as well as the requirement of DA system, on the other hand a graphical user interface needs to be developed at the substation side to monitor, control and supervise the system. If a DA system is to be built then MALTAB GUI would inadverantly be an important part of the system around with all the components and their specifications would be decided. Below given is the GUI created in Matlab , it consists of two main panels, one is the set point panel and other is the current value panel. As soon as the value in current value panel exceeds the values in the set point panel, a warning message is displayed to the operator by means of the status blocks changing its color, for the purpose of simulation the current value panel has been given pseudo random numbers which are generated as per the system clock from Matlab itself, in actual practice it is very convenient to build a hardware that can send the actual status of voltages and current at the distribution transformer.

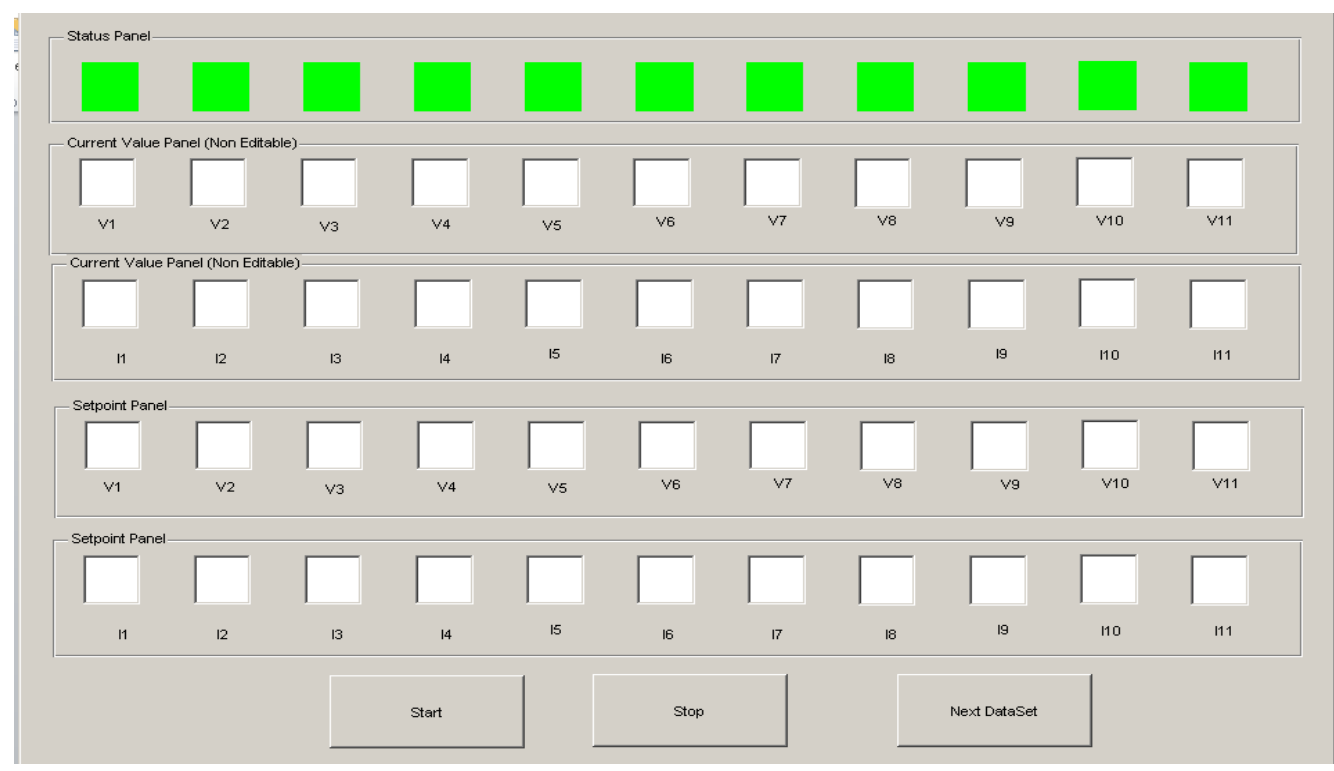

VIII. CONCLUSION

In this research article, a comprehensive approach toward distribution automation has been taken. Several methods were evaluated on the basis of cost and performance. In addition to that a new system based on Control Area Network has also been discussed. The proposed system is under development and GUI (graphical user interface) for the same has to be developed. Also all the technological solutions has been discussed keeping in view Indian scenario and development state in our country. Moreover the system proposed could be extended to incorporate many other important parameters of a distribution transformer such as oil temperature, the amount of level of oil in transformer etc.

\section{Acknowledgements}

We would like to express our sincere gratitude toward our alma mater institute Shriram Institute of Technology for providing us the required environment and resources to pursue our research work. 


\section{References}

[1] K. Clinard and John Redmon, Editors, Distribution Management Tutorial, IEEE PES Winter Meeting, Tampa, FL, February 1998.

[2] A. Pahwa and J.K. Shultis, Assessment of the Present Status of Distribution Automation, Report No. 238, Engineering Experiment Station, Kansas State University, Manhattan, KS, March 1992.

[3] D. Bassett, K. Clinard, J. Grainger, S. Purucker, and D. Ward, Tutorial Course: Distribution Automation, IEEE Publication 88EH0280-8-PWR.

[4] T.Moore, "Automating the Distribution Network," EPRI Journal, September 1984, pp. 22-28.

[5] T. Moore, J.B. Bunch, Guidelines for Evaluating Distribution Automation, EPRI Report EL-3728, November 1984.

[6] T. Kendrew, "Automated Distribution," EPRI Journal, January/February 1990, pp.46-48.

[7] J.B. Bunch, Guidelines for Evaluating Distribution Automation, EPRI Report EL-3728, November 1984.

[8] J.S. Paserba, N.W. Miller, S.T. Naumann, M.G. Lauby, and F.P. Sener, "Coordination of a Distribution Level Continuously Controlled Compensation Device with Existing Substation Equipment for Long Term Var Management," Paper No. 93 SM 437-4 PWRD, IEEE PES Summer Meeting, Vancouver, Canada, July 1993.

[9] G.T. Heydt, Electric Power Quality, Stars in a Circle Publications, West Lafayette, IN, 1991

[10] J. Douglas, "Power Quality Solutions,", IEEE Power Engineering Review, v. 14, no. 3, March 1994. 